\title{
Big data; sensor networks and remotely-sensed data for mapping; feature extraction from lidar
}

\author{
Lorato Tlhabano ${ }^{\mathrm{a}}$ \\ ${ }^{a}$ Department of Surveys and Mapping, Mapping Division, Cartography Section, Gaborone, South east, Botswana; \\ lrtlhabano@gov.bw
}

\begin{abstract}
Unmanned aerial vehicles (UAVs) can be used for mapping in the close range domain, combining aerial and terrestrial photogrammetry and now the emergence of affordable platforms to carry these technologies has opened up new opportunities for mapping and modeling cadastral boundaries. At the current state mainly low cost UAVs fitted with sensors are used in mapping projects with low budgets, the amount of data produced by the UAVs can be enormous hence the need for big data techniques' and concepts. The past couple of years have witnessed the dramatic rise of low-cost UAVs fitted with high tech Lidar sensors and as such the UAVS have now reached a level of practical reliability and professionalism which allow the use of these systems as mapping platforms. UAV based mapping provides not only the required accuracy with respect to cadastral laws and policies as well as requirements for feature extraction from the data sets and maps produced, UAVs are also competitive to other measurement technologies in terms of economic aspects. In the following an overview on how the various technologies of UAVs, big data concepts and lidar sensor technologies can work together to revolutionize cadastral mapping particularly in Africa and as a test case Botswana in particular will be used to investigate these technologies. These technologies can be combined to efficiently provide cadastral mapping in difficult to reach areas and over large areas of land similar to the Land Administration Procedures, Capacity and Systems (LAPCAS) exercise which was recently undertaken by the Botswana government, we will show how the uses of UAVS fitted with lidar sensor and utilizing big data concepts could have reduced not only costs and time for our government but also how UAVS could have provided more detailed cadastral maps.
\end{abstract}

Keywords: Big data, UAV, Sensor,Networks, Lidar, LAPCAS

\section{Introduction}

The expressions UAV (unmanned aerial Vehicles) photogrammetry (Eisenbeiss,2008, haarbrink 2007) describes a photogrammetric measurement platform, which operates remotely controlled, semi-autonomously, or autonomously, without a pilot physically present in the vehicle but controlling the UAV from the ground using a remote control system. UAVs used for mapping applications are equipped with a photogrammetric measurement system, including, but not limited to a small or medium size still-video or video camera, thermal or infrared camera systems, airborne LiDAR system, or a combination of these technologies. To orient themselves and to keep track of their current position(Martinez,2005) in flight UAVs use a number of sensors including but not limited to digital cameras, thermal or infrared camera systems, multispectral cameras, Global position system (including glonass), range camera sensors and altimeters. The application of these sensors in combination makes UAVs complete photogrammetric measurement tool opening up new exciting opportunities in cadastral surveying. The advent of UAVs has opened up new application in the close range domain by combining aerial and terrestrial photogrammetry with new near real time application and low - cost alternatives to the classical manned aerial photogrammetry. This is clearly illustrated on table 1 by Eisenbeiss.

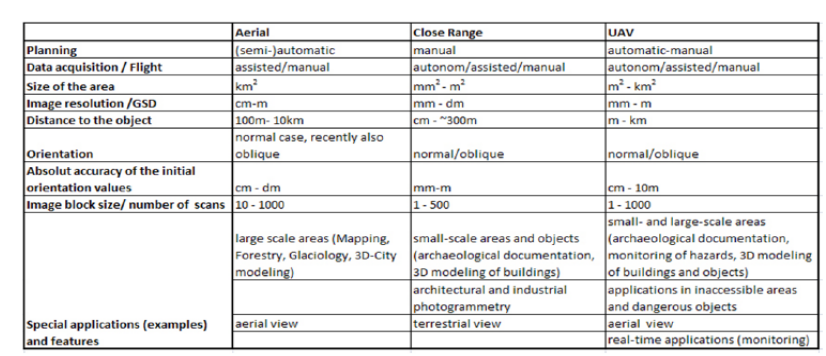

Table 1: Features of Aerial, close range and UAV photogrammetry (source Eisenbeiss, 2008)

The biggest advantage of UAVs manned aircraft is their ability to fly into difficult, inaccessible and remote terrain at relatively low cost. The incorporation of advanced sensors with smart software such as path planning software gives UAVs to operate autonomously further reducing operation costs by reducing the number of operators required on site. Addition of advanced sensors such as Lidar can lead to an enormous amount of cadastral data collected by UAVs more especial when mapping over a large area using more than one UAV. This data can take a long time to analyse or process, extract features or to develop an orthophoto map. This difficulty and complexity uploading, aggregating, and analyzing data across multiple platforms can be addressed by employing big data analytics.

(Abdulla, 2004) presented in the Manual of Photogrammetry the existing photogrammetric platforms, 
such as airborne, satellite and land-based platforms, as well as auxiliary systems. Thereby, the airborne platforms are subdivided in aircraft platforms such as single and twin engine aircrafts and other platforms like unmanned surveillance and reconnaissance aircraft systems, helicopters and balloons. Looking at aerial based platforms like, for example, kites, model helicopters, quadrotors1 and terrestrial based systems such as grains, rope-way, well-bucket and base bars, it is clear that not all platforms available for photogrammetric observations can be categorized using this classification. Furthermore, the category auxiliary systems do not represent the nonclassified platforms. This group remains for the positioning systems such as GPS, INS and stabilized platforms, as well as aerial survey flight management systems.

Kraus, 2007 focused in his book Photogrammetry: Geometry from images and Laser scans mainly on aerial photogrammetry. He does not explicitly categorize the platforms used in the aerial case. Chapter 3 (Kraus, 2007) concentrates however on photogrammetric recording systems and their applications, where the recording systems are classified into photochemical, photoelectronic, and digitizing systems. In addition, Kraus distinguishes between aerial and terrestrial cameras, while he separates terrestrial and mobile mapping methods.

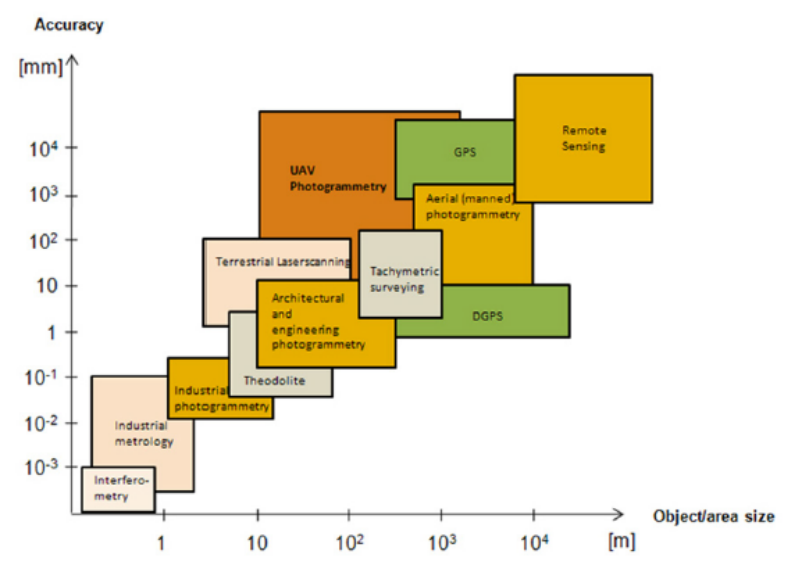

Figure 2: The accuracy of measurement methods in relation to the object/area size. Modified from Fig. 1.4 in Luhmann, et al., 2006, p. 4.

UAV-images can also be used for the high resolution texture mapping on existing DSMs and 3D-models, as well as for image rectification. The rectified images and derivates, like image mosaics, maps and drawings, can be used for image interpretation.

The implementation of GPS systems as well as the stabilization and navigation units allow precise flights, guaranteeing, on the one hand, sufficient image coverage and overlap and on the other hand, enabling the user to estimate the expected product accuracy before the flight.

Looking at rotary wing UAVs, the platform allows vertical take-off and landing negating the need for an available runway. Furthermore, the use of VTOL (Vertical take-off and landing) systems permits the image acquisition on a hovering point, while the camera is turning in vertical and horizontal direction which can be an especially useful figure when correcting or collecting missing data.

Airborne LiDAR systems, image-based systems only need the position and orientation at the time, where the image was acquired. Furthermore, it is still possible to georeference images with the indirect georeferencing method (bundle adjustment) using GCPs (Ground Control Points).

However, current developments in the field of UAV mapping focus also on the improvement of the GNSS and INS data by using DGPS (differential GPS; Bláha, 2011) and vision based navigation combined with inertial sensors, visual odometer and registration of a UAV onboard video (Conte and Doherty, 2008).

\section{Big Data}

The biggest advantage of traditional aerial mapping when using lidar sensors for cadastral mapping has been the ability of the aircraft to cover large areas of land with onboard processing equipment processing the data as its being collected. Low cost UAVs are in practice small and lightweight so the ability to cover large areas and carry sophisticated data collection equipment is negated. To make up for this deficiency UAVS have to

1) Work in pairs or teams

2) Transfer all the data they have collected to an outboard processing system

\section{Sensor Networking}

For the UAVS to work in pair or teams they have to possess a number of sensors which on top on enabling the UAV to maintain flight they have to enable them to communicate with each other. Sensor networks have to be designed to enable the UAVS to communicate and share and receive data. This data will then have to be transferred outboard for processing and analytics on specialised programs and equipment.

\section{Data Transfer}

UAVs are fitted with high speed modems such as 3G, 4G or WIFI to enable high speed data transfer (Sensefly,2015). Because of the various sensors on UAVs the amount of data collected is varied further increasing the complexity of the data collected. This data is only valuable if it can be processed and analysed in a timely fashion, therefore big data analytics are employed to extract the required sensor data from the data being transmitted. The data is then sent to the right program for processing e.g telemetry data can be sent to flight planning software while Lidar sensor data can be sent to GIS software for feature extraction. All this applications can be hosted in the cloud further reducing the cost of equipment required. The processing power of the cloud is an order of magnitude higher than that of traditional equipment meaning data processing can be done much quicker than on traditional equipment. Whereas a laptop computer running a GIS application can process data from a single drone in a couple of drones in a number of 
hours, an application hosted on the cloud can process data from multiple drones in a matter of minutes.

\section{LAPCAS}

The project for improvement (LAPCAS, 2009) of Land Administration Procedures, Capacity and Systems (LAPCAS) is an actor driven cooperation between Lantmateriet, the Swedish mapping, cadastre and Land Registration Authority and Ministry of Land Management Water and Sanitation Services in Botswana. The aim of the LAPCAS - Project is to accomplish long term solutions that are more effective at dealing with the question of land administration. It is hoped that this will result in social and economic development for Botswana as a nation.

The project consists of seven main goals which are stated as components, each dealing with different problems in land administration.

1) National systems for unique referencing of land parcels and location addresses

2)Improved land administration processes

3) A computerized Deeds Register

4) Procedures and legal support developed for systematic adjudication of rights in tribal land.

5) An organization that is competent to operate and maintain land administration and planning systems

6) A national spatial data infrastructure developed and implemented in planning and land administration systems

7) Training and study trips, dealing with training needs that are common for several components

In pursuit of the first goal the government (LAPCAS, 2010) with its partners first needed to register all register Land and their owner's country wide and then to survey and collect cadastral data for all allocated plots. This was done through the LAPCAS project under the National Land Registration (NLR) project. This was an enormous project that was outsourced to 41 private survey companies in 2016 alone at a cost of BWP 93, 661, 290.00 (approximately $\$ 8.5$ million). Some companies used aircraft fitted with Lidar sensors to speed up their survey.

\section{Conclusions}

The trend in UAV photogrammetry is moving towards the use of autonomous low cost UAVs working in teams(swarms) fitted with high speed internet modems and using Big Data analytics to process sensor data in real time. Countries and mapping organisations around the world would do well to take advantage of low cost UAV by adapting them to their needs and incorporating them into their everyday operations. Automation of the UAV flight and the improvements in the automation of the workflow discussed here for data processing, UAVs can now be applied for practical applications with real world impact. UAVS offer a cost effective way to collect cadastral data over large areas.

\section{Acknowledgements}

Firstly I would like to thank Ms Lucy Phalaagae(former Chief Surveyor with the Department) for her support and encouragement to write this paper, she believed in me even when I did not believe in myself, I would also like to thank the director Mr Oitsile for the immediate support he gave me once I told him about my paper, I would also like to acknowledge my head of division Mr Letlole who is always very diligent with what I write and generous with his advice, my other acknowledgements go out to Bashadi Seboni, Ephraim Kenneth, Annah Mogopodi, Mercy Lekone, and lastly I would like to thank the Government of Botswana and my Department for their generous support with their time and resources and their tireless quest for the progression of their employees.

\section{References}

Eisenbeiss, H., 2008. UAV photogrammetry in plant sciences and geology, In: 6th ARIDA Workshop on "Innovations in 3D Measurement, Modeling and Visualization, Povo (Trento), Italy.

Luhmann, T., Robson, S., Kyle, S. and Harley, I. (2006): Close Range Photogrammetry -Principles, Methods and Applications, Whittles Publishing, p.256

Verhoeven, G. J. J. (2009): Providing an archaeological bird's-eye view - an overall picture of ground-based means to execute low-altitude aerial photography (LAAP) in Archaeology, In: Archaeological Prospection, 16 (4), 233-243.

Haarbrink, R. B. and Koers, E., 2006. Helicopter UAV for photogrammetry and rapid response, In: International Archives of Photogrammetry, Remote Sensing and Spatial Information Sciences, ISPRS Workshop of Inter-Commission WG I/V, Autonomous Navigation, Antwerp, Belgium.

Haarbrink, R. B. and Eisenbeiss, H., 2008. Accurate DSM production from unmanned helicopter systems, In: International Archives of Photogrammetry, Remote Sensing and Spatial Information Sciences, ISPRS Congress, Beijing, China, XXXVII. Part B1, 12591264.

SenseFly, 2015. Angry birds at altitude, Tasked with a large and topographically challenging survey near Quito in Ecuador, AOC Ingeniería used an eBee for its data collection. But the terrain wasn't the only problem the team face. Page $1-4$

Herwitz, S., L. Johnson, et al. (2004): "Demonstration of UAV-based imaging for agricultural surveillance and decision support." Comput. Electron, Agr. 44: 49-61.

Bláha, M., Eisenbeiss, H., Grimm, D. and Limpach, P. (2011): Direct Georeferencing of UAVs, In: The International Archives of the Photogrammetry, Remote Sensing and Spatial Information Sciences, UAV-g congress 2011, accepted.

Nagai, M., Shibasaki, R., Manandhar, D. and Zhao, H. (2004): Development of digital surface and feature extraction by integrating laser scanner and CCD sensor with IMU, In: International Archives of the 
Photogrammetry, Remote Sensing and Spatial Information Sciences, XX ISPRS Congress, Istanbul, Turkey, XXXV-B5, 655-659.

D. Shim, D., Chung, H., Kim, H. J. and Sastry, S., 2005: Autonomous exploration in unknown urban environments for unmanned aerial vehicles, In: AIAA GN\&C Conference, San Francisco, California.

LAPCAS (2009): Improvements of land administration procedures, Capacity and Systems in Botswana, Project description, Lantmateriet in Sweden and Ministry of Lands and Housing in Botswana

LAPCAS (2010): Guidelines for the systematic adjudication process (Pilot Project in Matebele) April 2010 